\title{
Stimulus preference and the transitivity of preference
}

\author{
TERRY W. BELKE \\ Harvard University, Cambridge, Massachusetts
}

\begin{abstract}
Four pigeons were exposed to multiple schedules with concurrent variable interval (VI) components and then tested for preference transfer. Half of the pigeons were trained on a multiple concurrent VI 20-sec, VI 40-sec/concurrent VI 40-sec, VI 80-sec schedule. The remaining pigeons were trained on a multiple concurrent VI 80-sec, VI 40-sec/concurrent VI 40-sec, VI 20-sec schedule. After stability criteria for time and response proportions were simultaneously met, four preference transfer tests were conducted with the stimuli associated with the VI 40-sec schedules. During the transfer tests, each pigeon allocated a greater proportion of responses $(M=0.79)$ and time $(M=0.82)$ to the stimulus associated with the VI 40-sec schedule that was paired with the VI 80-sec schedule than to the VI 40-sec schedule stimulus paired with the VI 20-sec schedule. Absolute reinforcement rates on the two VI 40-sec schedules were approximately equal and unlikely to account for the observed preference. Nor was the preference consistent with the differences in local reinforcement rates associated with the two stimuli. Instead, the results were interpreted in terms of the differential value that stimuli acquire as a function of previous pairings with alternative schedules of reinforcement.
\end{abstract}

When a pigeon is confronted with a choice between two alternatives, each of which was previously experienced in conjunction with other alternatives, how does the pigeon's prior experience influence the pigeon's distribution of behavior between these alternatives? Specifically, what factors related to the pigeon's prior experience guide the choice behavior?

Previous research suggests that differences in prior reinforcement probability (Herrnstein \& Loveland, 1976) and absolute reinforcement rates, but not local reinforcement rates (Williams \& Royalty, 1989), influence the distribution of time and behavior to novel stimulus pairings. Herrnstein and Loveland initially trained pigeons on four different schedules of reinforcement, each associated with a different colored response key. Following this initial training, pigeons were exposed to four of the six possible concurrent pairs of the four schedules. After extensive training, preference probes were conducted with the two remaining schedule combinations.

Results showed that preferences in the transfer tests overmatched relative rates of reinforcement from the training pairs. On the basis of these results, Herrnstein and Loveland (1976) suggested that "novel pairings within the network are treated as if they were on ratio schedules until proven otherwise by an ongoing interaction between

The author wishes to thank Jamie Taylor, Linda Anderson, William Reynolds, Jr., William Vaughan, Jr., Richard J. Herrnstein, and Gene M. Heyman for their contributions to this research and their comments on an earlier manuscript. Correspondence should be sent to T. W. Belke, Department of Psychology, Harvard University, William James Hall, 33 Kirkland Street, Cambridge, MA 02138 (e-mail: twb@harvunxw). reinforcement and responding" (p. 152). Thus, before reinforcement was obtained, the pigeon responded to the novel stimuli as if they were ratio schedules, and choice was governed by perceived differences in reinforcement probability.

In a series of experiments, Williams and Royalty (1989) concluded that absolute, but not local, rates of reinforcement accounted for preference transfer to novel stimulus combinations. In their first experiment, pigeons were trained on multiple schedules with concurrent variable interval (VI) components, and then probe tests of novel stimulus pairings were conducted. For one pairing, absolute and local rates of reinforcement predicted preference for the same stimulus in transfer tests. Transfer tests confirmed this prediction. However, in a second transfer test, absolute and local reinforcement rates predicted opposite preferences during transfer tests. Results showed slight or no preference for either stimulus. Thus, neither of the predictions, based on absolute and local reinforcement rates, was supported.

A replication of this condition using a discrete trials procedure, in which absolute and local reinforcement rates made opposite predictions, produced a moderate preference consistent with the prediction based on absolute rather than local reinforcement rates. In a final experiment, Williams and Royalty (1989) used a discrete trial procedure in which predictions based on absolute reinforcement rates and local reinforcement probabilities differed. The results were again inconsistent with control by the local reinforcement probabilities. On the basis of the results from these three experiments, Williams and Royalty concluded that absolute rather than local reinforcement rates control preference transfer to novel con- 
texts and that, contrary to the melioration theory of choice (Herrnstein \& Vaughan, 1980; Vaughan, 1985), local reinforcement rates do not govern choice.

Edmon, Lucki, and Grisham (1980) also suggested that absolute rates of reinforcement controlled preference transfer. Pigeons were exposed to a sequence of multiple schedules. In each multiple schedule, one schedule was associated with a red key and the other with a green key. After responding had stabilized on each schedule, test sessions with transfer probes were conducted. Results showed that for the multiple schedules, relative response rates undermatched relative reinforcement rates. However, for the transfer tests, preference overmatched relative reinforcement rates. The alternative with the higher absolute rate of reinforcement was strongly preferred.

In summary, these studies suggest that differences in absolute rates of reinforcement, or possibly reinforcement probability, control preference transfer from prior choice contexts to a novel context. However, these studies neglect differential stimulus value as a factor in preference transfer. Guttman (1959) proposed that stimuli acquire value as a function of the schedules of reinforcement with which they are associated.

This principle can also be applied to the larger context in the following way. If two stimuli are associated with the same schedules of reinforcement, then they would have the same value; however, the value of these stimuli may also vary as a function of the schedules of reinforcement provided by an alternative in a choice context. In essence, I propose that a stimulus acquires a value that depends upon the relationship of the rate of reinforcement associated with that stimulus to the rate of reinforcement provided by an alternative and that these differential stimulus values influence time and response allocation in preference transfer tests. Thus, the purpose of the present experiment was to investigate the transfer of preference to a novel stimulus pairing where both stimuli had the same programmed rate of reinforcement in previous pairings with different schedules of reinforcement.

On the basis of this explanation, one would predict that a VI 40 -sec schedule paired with a VI 20 -sec schedule should acquire a low value, while a VI $40-\mathrm{sec}$ schedule paired with a VI 80 -sec schedule should acquire a high value. If a transfer test is conducted using the stimuli associated with these two VI 40 -sec schedules, then a preference for the VI 40-sec schedule stimulus that was paired with the VI 80-sec schedule would be predicted.

\section{METHOD}

\section{Subjects}

Four pigeons with extensive experimental histories served as subjects. The birds were kept at $80 \%$ of free-feeding weight throughout the course of the study. Supplemental feeding was given after experimental sessions when needed to maintain the target weight.

\section{Apparatus}

An operant-conditioning chamber equipped with five keys was used for this experiment. The chamber was $53 \mathrm{~cm}$ deep, $51 \mathrm{~cm}$ wide, and $30 \mathrm{~cm}$ long and located within a plywood shell insulated with foam rubber to reduce extraneous noise. Response keys were $2 \mathrm{~cm}$ in diameter, $6 \mathrm{~cm}$ apart, center to center, and $22 \mathrm{~cm}$ above the wire-mesh floor. Each response key could be transilluminated from the rear and required $1.5 \mathrm{~N}$ of force to operate. Only four of the five keys were used in the present experiment. The rightmost key was inoperative.

Reinforcement was $4 \mathrm{sec}$ access to a milo and wheat mix. The feeder opening was $5 \mathrm{~cm}$ wide, $34 \mathrm{~cm}$ long, and located $5 \mathrm{~cm}$ above the wire-mesh floor. An electric motor raised and lowered the feeder. The feeder was illuminated and the keylights and houselights were extinguished during reinforcement. The chamber was illuminated by two white lights mounted on the sides of the chamber $4 \mathrm{~cm}$ from the top. A ventilation fan masked noise and circulated air within the chamber. A PDP-8A computer (Digital Equipment) controlled experimental events and recorded data.

\section{Procedure}

Pigeons 544 and 525 were randomly assigned to Group 1, and Pigeons 547 and 435 were assigned to Group 2. Each group of birds was exposed to a different multiple schedule with concurrent VI components. Table 1 outlines the multiple schedules given to Groups 1 and 2. Each component of a multiple schedule operated for $1 \mathrm{~min}$. Components were alternated, and there was a 10-sec blackout between transitions. For example, Keys 1 and 2 would be operative for $1 \mathrm{~min}$, then there was a 10 -sec blackout, followed by Keys 3 and 4 becoming operative. In each component, a 1-sec changeover delay was in effect to prevent direct reinforcement of switching behavior. Independent VI schedules with approximately exponential interval distributions (Fleshler \& Hoffman, 1962) were programmed for each key.

A session was defined by 15 exposures to each component in the multiple schedule. The order of presentation of components alternated across successive days. On one day, the session would begin with Component $\mathrm{A}$ and end with Component $\mathrm{B}$, and on the next day, the session would begin with Component $B$ and end with Component A.

This training phase remained in effect until a stability criterion was met at least once for each component. After 15 sessions, response and time proportions for the 9 preceding sessions were divided up into blocks of 3 sessions. The means for the blocks were calculated and compared to determine stability. Preference was judged stable when the means for the three blocks differed by no more than 0.05 and there was no downward or upward trend in either the response or time means. Sessions were run 7 days a week, and the training phase remained in effect until a bird simultaneously met the stability criteria for both time and response allocation in both components.

During the transfer test phase, 1-min exposures to the Key 2 and Key 3 combination were programmed to occur between exposures to the components of the multiple schedule. During each transition between components, a random selection from a list determined if a transfer test probe would occur. Five transfer test probes occurred during each transfer test session. Extinction was in effect for the transfer test key combination. Responses, changeovers, and time allocation were recorded during the test exposures. Transfer

Table 1

Outline of the Multiple Schedules Given to Groups 1 and 2

\begin{tabular}{|c|c|c|c|c|}
\hline \multirow[b]{2}{*}{ Key Color } & \multicolumn{2}{|c|}{ Component A } & \multicolumn{2}{|c|}{ Component B } \\
\hline & $\begin{array}{l}\text { Key } 1 \\
\text { White }\end{array}$ & $\begin{array}{c}\text { Key } 2 \\
\text { Red }\end{array}$ & $\begin{array}{l}\text { Key } 3 \\
\text { Green }\end{array}$ & $\begin{array}{c}\text { Key } 4 \\
\text { Yellow }\end{array}$ \\
\hline $\begin{array}{l}\text { Group 1 } \\
\text { Group } 2\end{array}$ & $\begin{array}{l}\text { VI } 20-\mathrm{sec} \\
\text { VI } 80-\mathrm{sec}\end{array}$ & $\begin{array}{l}\text { VI } 40-\mathrm{sec} \\
\text { VI } 40-\mathrm{sec}\end{array}$ & $\begin{array}{l}\text { VI } 40-\mathrm{sec} \\
\text { VI } 40-\mathrm{sec}\end{array}$ & $\begin{array}{l}\text { VI } 80-\mathrm{se} \\
\text { VI } 20 \text {-se }\end{array}$ \\
\hline
\end{tabular}

Note-Transfer tests used the red and green stimuli associated with Keys 2 and 3. 
test sessions were followed by two baseline sessions. A total of four transfer test sessions were conducted with each pigeon.

\section{RESULTS}

Figures $1 \mathrm{~A}$ and $1 \mathrm{~B}$ show response, time, and reinforcement proportions obtained for each bird on the VI 40-sec schedules during the training phase. Proportions were calculated from the nine sessions that met the stability criterion. In general, time proportions were more consistent with the obtained rates of reinforcement in the two components for both groups than were response proportions.

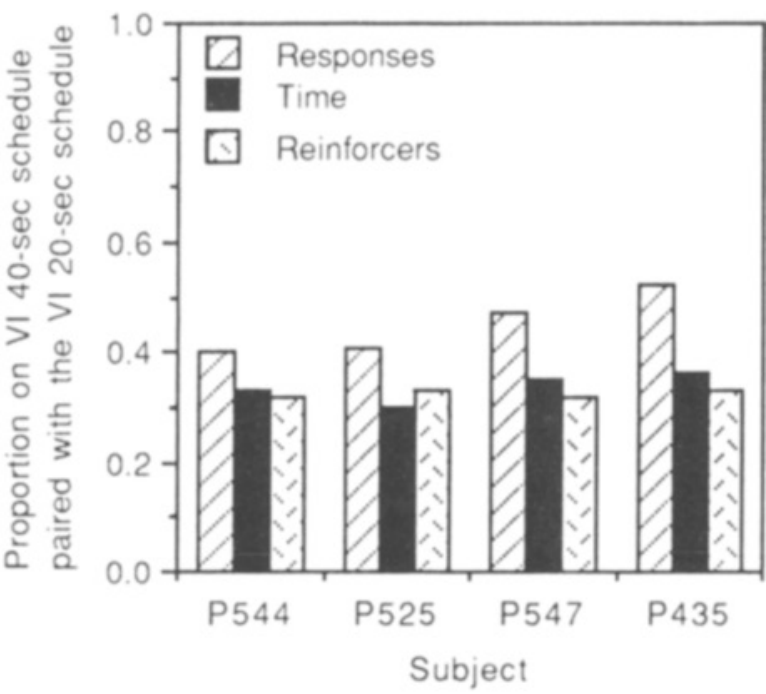

(B)

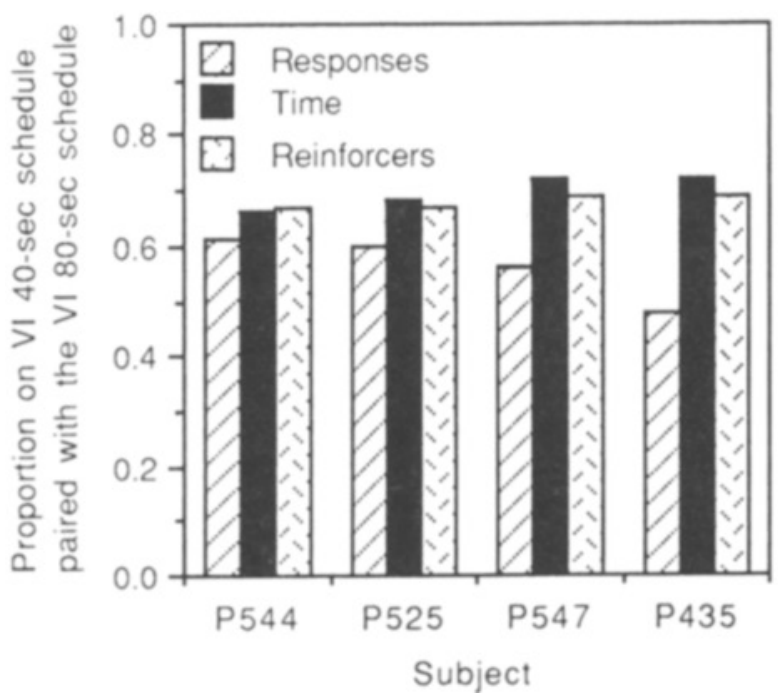

Figure 1. Mean response, time, and reinforcement proportions on the VI 40-sec schedules in each component during the training phase.

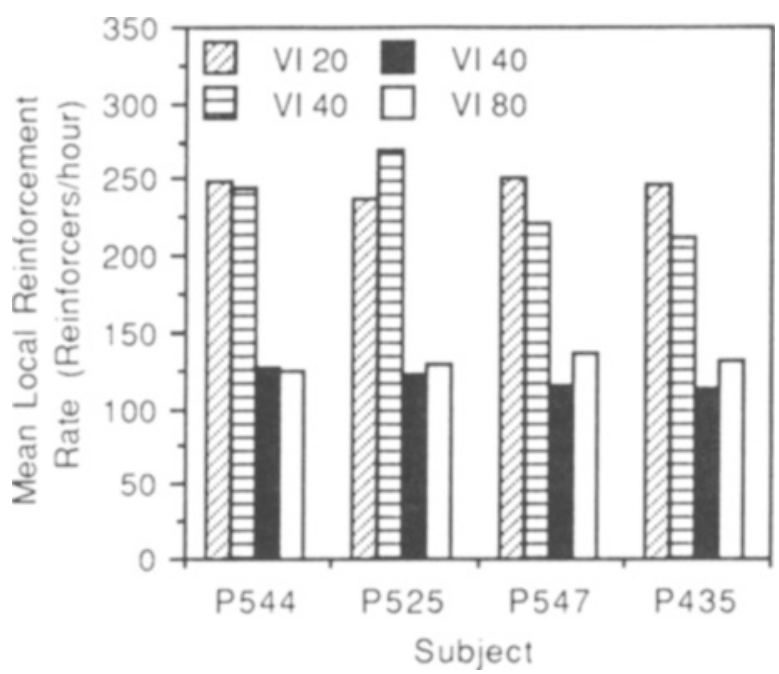

Figure 2. Mean local reinforcement rates by schedule value in each component for each pigeon during the training phase.

For the concurrent VI 20-sec/VI 40-sec component (Figure 1A), across both groups, the average time, response, and reinforcement proportions on the VI 40-sec schedule were $0.33,0.45$, and 0.32 , respectively. For the concurrent VI 40-sec/VI 80-sec component (Figure 1B), average time, response, and reinforcement proportions on the VI 40 -sec schedule were $0.70,0.56$, and 0.68 , respectively.

Pigeon 435 in Group 2 showed the greatest inconsistency between response, time, and reinforcement allocations. For this pigeon, time allocations matched reinforcement allocations; however, response allocations showed indifference. This difference in the consistency of time and response allocations with reinforcement allocation has been noted before. Baum (1979), in a review of matching studies, found that time allocations provide closer approximations to matching than response allocations.

Figure 2 shows the mean local reinforcement rates for each subject for the nine stability sessions prior to the introduction of the transfer tests. Local rates of reinforcement were calculated as the number of reinforcements obtained from an alternative divided by the time spent on that alternative.

Mean local rates of reinforcement for the VI 20-sec, VI 40-sec, VI 40-sec, and VI 80-sec schedules for both groups were 246, 236, 120, and 131 reinforcers per hour, respectively. Thus, the local rate of reinforcement on the VI 40-sec schedule associated with the VI 20-sec schedule ( $M=236$ reinforcers/hour) was higher than for the VI 40 -sec schedule associated with the VI 80 -sec schedule $(M=120$ reinforcers/hour). This difference reflects the levels of reinforcement at equilibrium within the respective contexts of the two components. The mean local rate of reinforcement in the two components differed by approximately 116 reinforcers per hour at equilibrium.

Figure 3 shows the mean absolute reinforcement rates for each subject during the training phase. Mean abso- 


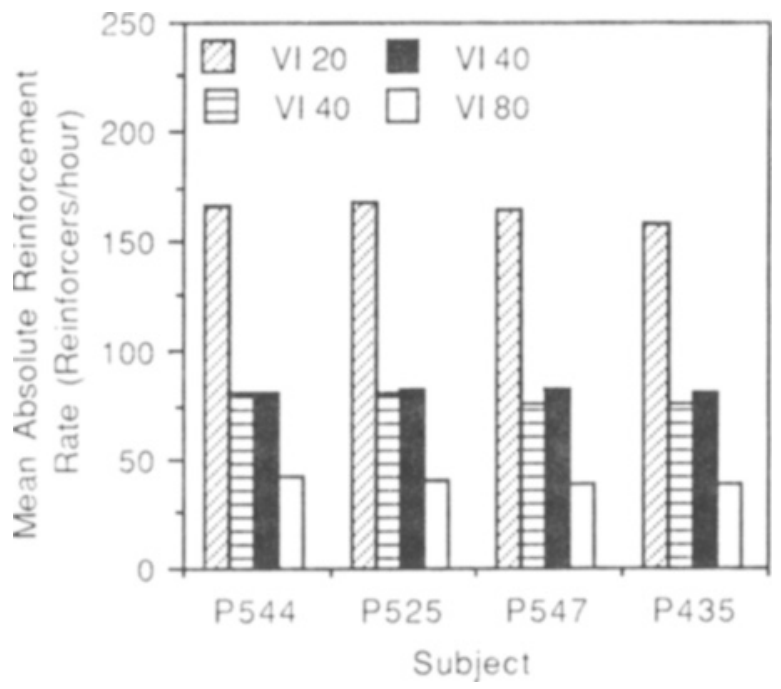

Figure 3. Mean absolute reinforcement rates by schedule value in each component for each pigeon during the training phase.

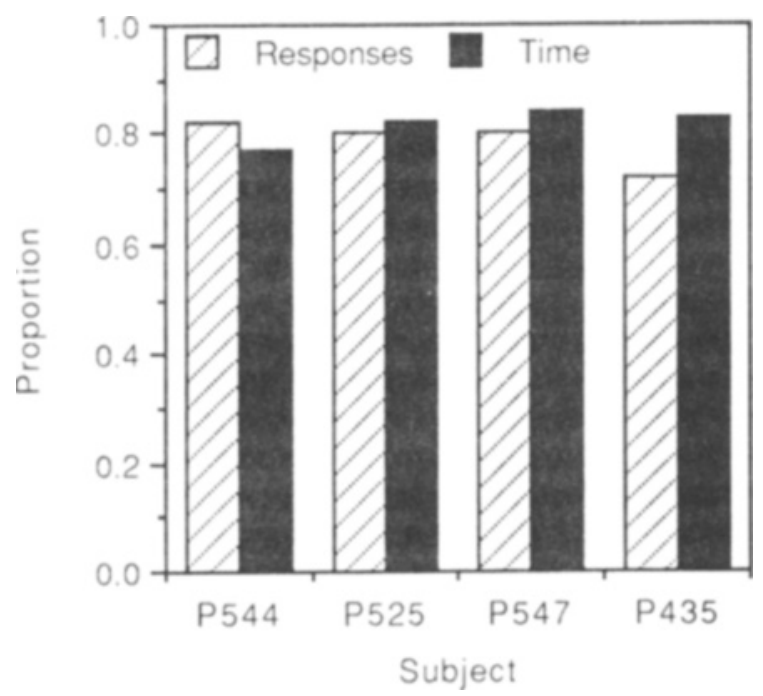

Figure 4. Mean response and time proportions during the transfer test for the stimulus associated with the VI 40-sec schedule that was paired with the VI 80-sec schedule during the training phase. lute reinforcement rates were calculated as the number of reinforcements obtained from an alternative divided by the total session time and averaged over the nine stability sessions. Figure 3 shows that the mean absolute reinforcement rates were approximately the same for both VI 40 sec schedules. Mean absolute reinforcement rates for the VI 20 -sec, VI 40-sec, VI 40-sec, and VI 80-sec schedules were $164,78,83$, and 40 reinforcers per hour. Overall, the mean absolute reinforcement rate on the VI 40 -sec schedule associated with the VI 20 -sec schedule averaged 78 reinforcers per hour compared with 83 reinforcers per hour for the VI 40-sec schedule associated with the VI 80-sec schedule. Thus, the two VI 40-sec schedules differed by only 5 reinforcers per hour.

Figure 4 shows the mean response and time allocations during transfer tests to the stimulus associated with the VI 40-sec schedule paired with the VI 80-sec schedule during training. On average, the proportion of responses allocated to this stimulus was 0.79 , while the proportion of time was 0.82 . Table 2 shows response and time proportions for each individual subject.

Table 2 also compares the observed transfer test response and time allocations on the VI 40-sec key that was associated with the VI 80-sec schedule in training to predictions based on relative absolute reinforcement rates, relative local reinforcement rates, and relative reinforcement probability. Reinforcement probabilities were calculated from the training phase by dividing the number of reinforcements by the number of pecks for each key and expressing the values relatively. A comparison is also made with the time and response observed for that key during training sessions.

The response and time allocations to that key during the transfer tests were 0.79 and 0.82 , respectively. Relative absolute reinforcement rates predicted response and time allocations of 0.52 on average for this key. Relative local reinforcement rates predicted allocations of 0.34 on average for this key. Finally, relative reinforcement probabilities predicted average allocations of 0.55 for time and responses. Thus, predictions based on absolute reinforcement rates, local reinforcement rates, and reinforcement probabilities during training do not account for the response and time allocations observed during the transfer tests.

Table 2

Predictions for Transfer Test Time and Response Proportions

Based on Relative Absolute Reinforcement Rates, Relative Local

Reinforcement Rates, Relative Reinforcement Probabilities for the

VI 40-Sec Key that was Associated with the VI 80-Sec Schedule

in the Training Phase, and Time and Response Proportions

Obtained During the Training Sessions and Transfer Tests

\begin{tabular}{|c|c|c|c|c|c|c|c|}
\hline \multirow[b]{2}{*}{ Pigeon } & \multirow{2}{*}{$\begin{array}{l}\text { Relative } \\
\text { Absolute }\end{array}$} & \multirow{2}{*}{$\begin{array}{c}\text { Relative } \\
\text { Local }\end{array}$} & \multirow{2}{*}{$\begin{array}{c}\text { Relative } \\
\text { Probability }\end{array}$} & \multicolumn{2}{|c|}{$\begin{array}{c}\text { Observed } \\
\text { Training } \\
\text { Proportions } \\
\end{array}$} & \multicolumn{2}{|c|}{$\begin{array}{c}\text { Observed } \\
\text { Transfer } \\
\text { Proportions }\end{array}$} \\
\hline & & & & Response & Time & Response & Time \\
\hline 544 & 0.51 & 0.34 & 0.49 & 0.61 & 0.66 & 0.82 & 0.77 \\
\hline 525 & 0.51 & 0.31 & 0.45 & 0.60 & 0.68 & 0.80 & 0.82 \\
\hline 547 & 0.52 & 0.34 & 0.61 & 0.56 & 0.72 & 0.80 & 0.84 \\
\hline 435 & 0.52 & 0.35 & 0.64 & 0.48 & 0.73 & 0.72 & 0.83 \\
\hline Mean & 0.52 & 0.34 & 0.55 & 0.56 & 0.72 & 0.79 & 0.82 \\
\hline
\end{tabular}


A comparison of time and response allocations to this key during training and transfer phases shows that the assumption that the subjects merely generalize allocations from these keys during training accounted for the transfer test allocations better than did consideration of absolute reinforcement rates, local reinforcement rates, and reinforcement probabilities. During training sessions, on average, the response proportion for this key was 0.56 , while the equivalent proportion during the transfer tests was 0.79 . For time allocation, the average proportion was 0.72 for training sessions and $\mathbf{0 . 8 2}$ for transfer tests. For both response and time proportions, allocations during transfer tests were significantly greater than during training $[F(1,3)=465, p<.001$, and $F(1,3)=189$, $p<.001$, respectively]. These results suggest that the time and response proportions allocated to this key during the transfer test are a function of responding on these keys during training but that the allocations during transfer were not merely a generalization of the proportions during the training sessions. Response and time allocations during the transfer tests were more extreme than would be expected on the basis of allocations to these keys during the training sessions.

\section{DISCUSSION}

The present experiment showed that, in the absence of any difference in absolute rates of reinforcement, preference transfers in accord with differential stimulus preferences. The pigeons displayed a marked preference for the stimulus that was associated with a schedule of reinforcement that had been preferred in previous pairings over a stimulus that was associated with the same schedule of reinforcement but had not been preferred in previous pairings. The observed preference was not a function of either absolute or local rates of reinforcement. Absolute reinforcement rates were approximately equal for the two stimuli in the training components and therefore could not account for the preference observed in the transfer tests. Nor was the observed preference consistent with the difference in local rates of reinforcement between the two stimuli in the training components. In addition, the preferences were greater than would be expected on the basis of generalization of response and time allocations from the training components.

Williams and Royalty (1989) concluded that absolute rather than local rates of reinforcement account for preference transfer. The present experiment showed that a difference in absolute rates of reinforcement between the schedules associated with the stimuli used in transfer tests is not necessary to produce preference transfer. Preference transfer may be a function of a difference in absolute reinforcement rates when such a difference exists. However, in the experiments of Williams and Royalty, the effects of stimulus preference and absolute reinforcement rates were confounded.

A point of contention remains as to whether or not these transfer tests function as a test of melioration, and the issue is the nature of local rates of reinforcement (Herrnstein, 1991). First, local reinforcement rates are clearly more sensitive to context than are absolute reinforcement rates. Absolute rates of reinforcement are approximately the same both within and outside a choice context. In contrast, local rates of reinforcement vary considerably depending upon the context and may not exist outside of a choice context. For example, the VI 40 -sec schedule in the present experiment had the same absolute rate of reinforcement of approximately 80 reinforcers per hour in both components, but the local rates of reinforcement in the two components at equilibrium differed by around 116 reinforcers per hour. Thus, local rates of reinforcement are more context sensitive than are absolute rates, and this may account for the difference in generalizability between absolute and local rates of reinforcement. If local rates of reinforcement are less generalizable or do not generalize to a novel context, the validity of the transfer tests as a test of melioration is called into question.

Second, local rates of reinforcement, as calculated on session-level data, describe the outcome of an underlying process at equilibrium rather than functioning as the actual process. A high local rate of reinforcement can occur on an alternative with a low absolute rate of reinforcement simply as a function of the subject spending most of its time on the other schedule. To suggest that this high local rate of reinforcement draws an animal toward the alternative in a novel context does not seem sensible since the high rate is a function of the animal largely ignoring that schedule. In addition, the results of the present study suggested that subjects tend to allocate time and responses to stimuli in a novel context in accord with allocations in their previous context. Overall, these transfer tests challenge our conception of melioration, but their validity as tests of melioration is open to question.

Herrnstein and Loveland (1976) suggested that pigeons initially respond to the novel stimuli as if they were associated with concurrent variable ratio/variable ratio schedules. Rather than matching, pigeons tended to show exclusive preference for the alternative with the higher valued schedule. In the present experiment, the pigeons also overmatched relative reinforcement probabilities but did not respond exclusively to the alternative with the higher reinforcement probability. Relative reinforcement probabilities did not account for the time and response preferences observed during the transfer tests. Thus, the results from the present experiment, like the results from Williams and Royalty (1989), do not support the contention that pigeons respond to novel pairings of stimuli as if they were on concurrent ratio schedules.

The results from the present study, along with the results from a pilot study, suggest that the function relating stimulus preference in transfer tests to differential histories of schedule pairing is not a step function. In the present study, the stimulus that was preferred during the transfer tests was associated with a schedule (VI 40-sec) that set up reinforcement twice as often as the schedule it was paired with during the training sessions (VI 80- 
sec). The other stimulus was associated with the same schedule (VI 40-sec) during training, but it was paired with a schedule (VI 20-sec) that set up reinforcement twice as often. Preference for the former stimulus was around 0.80 during transfer tests. In the pilot study, the equivalent schedules were VI 180 -sec, VI 60 -sec, VI 60 -sec, and VI 20-sec. In this pilot study, the stimulus associated with the VI 60-sec schedule that was paired with a VI 180sec schedule during training was almost exclusively preferred during transfer tests over the stimulus associated with the VI 60-sec schedule that was paired with the VI 20 -sec schedule. Taken together, the results from the present study and this pilot study suggest that the greater the difference in preference histories, the more likely the observed performance will appear to be like performance on concurrent variable ratio/variable ratio schedules.

Although the present study demonstrates that stimulus preferences can play a role in preference transfer, the interaction of stimulus preference with differences in absolute reinforcement rates remains to be investigated. Understanding this interaction will further clarify preferences observed in novel contexts.

\section{REFERENCES}

BAUM, W. M. (1979). Matching, undermatching, and overmatching in studies of choice. Joumal of the Experimental Analysis of Behavior, 32, 269-281.

EDMON, E. L., LuCKI, 1., Grisham, M. G. (1980). Choice responding following multiple schedule training. Animal Learning \& Behavior, 8, 287-292.

Fleshler, M., \& HoffMan, H. S. (1962). A progression for generating variable-interval schedules. Journal of the Experimental Analysis of Behavior, 5, 529-530.

GuTrMAN, N. (1959). Generalization gradients around stimuli associated with different reinforcement schedules. Journal of Experimental Psychology, 58, 335-340.

Herrnstein, R. J. (1991). Reply to Binmore and Staddon. American Psychologist, 46, 799-801.

HerRnstein, R. J., Loveland, D. H. (1976). Matching in a network. Journal of the Experimental Analysis of Behavior, 26, 143-153.

HerRnStein, R. J., \& VAUGHAN, W. (1980). Melioration and behavioral allocation. In J. E. R. Staddon (Ed.), Limits to action (pp. 143-176), New York: Academic Press.

VAUGHAN, W., JR. (1985). Choice: A local analysis. Journal of the Experimental Analysis of Behavior, 43, 383-405.

Williams, B. A., Royalty, P. (1989). A test of the melioration theory of matching. Journal of Experimental Psychology: Animal Behavior Processes, 15, 99-113.

(Manuscript received July 21, 1989;

revision accepted for publication May 11, 1992.) 